\title{
Application of UAV Remote Sensing Mapping in Map Drawing
}

\author{
Xu Chen, Kuan He, Yuntong Liu \\ Yellow River Conservancy Technical Institute, Kaifeng, Henan, China
}

\begin{abstract}
UAV aerial remote sensing system has the characteristics of strong real-time, flexible, high image resolution and low cost, which can be applied to map mapping tasks under various terrain. In this paper, the key technology of UAV Remote Sensing Surveying and mapping, the process of image processing, the research of mosaic method and the field application of remote sensing technology are studied. Aiming at the characteristics of UAV image with high resolution and small image frame, three methods of image map making are proposed, namely, single image geometric correction method, mosaic correction method and aerial triangulation method. This paper focuses on the key technical problems of the three methods, and makes a comprehensive analysis and experimental verification of each method from the aspects of mapping effect, accuracy and efficiency. The experimental results show that the UAV remote sensing technology can meet the real-time basic surveying and mapping data requirements of urban mapping. This method can meet the needs of 1:500 high-precision mapping. The system can reduce the cost and improve the usability when it is used to update the basic data of Urban Surveying and mapping.
\end{abstract}

Keywords: UAV, aerial remote sensing, basic mapping data, map mapping.

\section{Introduction}

Topographic mapping is a highly targeted work, but also an important work in the construction project. UAV aerial photogrammetry technology plays a great role in topographic mapping [1-2]. In the use of UAV measurement technology, on the one hand, it reflects the flexibility of the technology, making the aerial photography broader, on the other hand, it also reduces the cost of capital, so as to meet the requirements of many topographic mapping. In addition, from another point of view, UAV aerial photogrammetry technology is a new scientific measurement technology developed by computer technology, digital communication technology and $3 \mathrm{~S}$ technology, which is a full complement in the field of aerial remote sensing [3]. Therefore, UAV aerial photogrammetry is used more and more widely.

UAV aerial photogrammetry is widely used, mainly depends on the following characteristics of UAV aerial photogrammetry technology [4-6]:

(1) The measurement of buildings is accurate, relative to satellite mapping, UAV can save time, faster, more agile to provide users with the required measurement information in time, and the measurement price of UAV is relatively low. In addition, UAV measurement work is widely used. Compared with manual surveying and mapping, UAV measurement system is more prominent in any aspect, and has strong comprehensive application ability. UAV measurement system can not only play its role alone, but also combine with satellite rocker aerial surveying and mapping and ground survey means;

(2) The application condition of UAV aerial survey technology is very convenient. From the perspective of UAV aerial survey, it usually works under low altitude conditions, and is not affected by the climate too much. At the same time, it does not have high requirements for the site. There is a relatively stable place to realize the take-off and landing process. It's easy to operate. It takes about 15 minutes to take off. It's also very helpful to the transportation industry. Based on the daily measurement task, it can measure tens to hundreds of square kilometers of aerial survey information every day;

ISSN: 0010-8189 
(3) In the process of UAV aerial photogrammetry, the surface data can be collected accurately and quickly. At the same time, the UAV aerial photogrammetry system has the functions of color digital photography and camera, which makes the acquisition of surface data more accurate and agile, and can generate three-dimensional Orthophoto Image Map and three-dimensional visual data. These data are able to realize the engineering application An important factor in the process. In addition, UAV aerial photogrammetry technology is also very powerful in the comprehensive ability of Surveying and mapping, it can work with aerial surveying and mapping and satellite remote sensing at the same time, so as to effectively and accurately obtain the measurement information and data. Compared with satellite and man-machine mapping, it can quickly complete the work in a limited time, and provide customers with the required data.

\section{Analysis and problem description of urban green landscape pattern}

In order to make the work of aerial triangulation get comprehensive and good development, the first thing to do is to determine the process before doing the work of triangulation. In the process of calculating aerial triangulation, there are many kinds of systems and software, including high-resolution remote sensing image integrated mapping system, beam adjustment software and so on [7]. In the process of system software application, we should do the following work: first, prepare the corresponding data, set the original data format to IPG format, check all the data used, including the focal length and coordinates of the camera, and make clear the aerial data and control point coordinate file; second, correct the distortion difference, starting from the UAV aerial photography From the point of view, all the non measurement cameras are used, and the relative distortion difference is large, and the photo has the problem of edge distortion, so we can correct the program through the distortion method; third, build the elevation file of the survey area, and combine the influence of distortion correction according to the relevant parameters, so as to effectively complete the automatic internal orientation, and further determine the initial offset of the flight belt In the process of automatic relative orientation, we avoid some basic matters and ensure that the number of relative orientation points is greater than 900 on the photo, so as to achieve the accuracy of distribution.

In the work of digital line drawing, the full digital photogrammetric workstation is used, and the reasonable editing work is carried out under the image editing software [8]. The correct format is the DWG format. During the work, we must pay attention to the following matters: first, in the process of mapping, we need to build the ground model corresponding to the number, and then determine the direction, There will be a certain degree of difference, because the manual method of automatic interactive operation, so, according to the operation process to carry out reasonable work, we must abide by the procedures at the same time, improve the vigilance, so that the degree of error is controlled within the specified range; second, before mapping, we must first ensure the accuracy of positioning, and each factor has a correct color, color, color, color, etc Third, professional training should be given to the mapping staff to minimize the error of manual collection of ground features and landforms. In addition, for the elements that are blocked due to various reasons, corresponding countermeasures should be made to remedy them in a timely manner.

This paper is mainly reflected in the construction of the classification system of urban green landscape from the perspective of spectral analysis, and puts forward the method of spectral green corridor division based on the combination of green patches. In the design of urban landscape architecture, it is necessary to build urban green landscape and design urban landscape architecture by selecting excellent lawn construction, shrub planting, plant community construction, etc., so as to form a pattern of urban landscape, improve the greening volume ratio of the city, improve the ecological environment, and achieve the goal of urban livability. The analysis case of urban green landscape pattern is shown in Figure 1.

ISSN: 0010-8189 


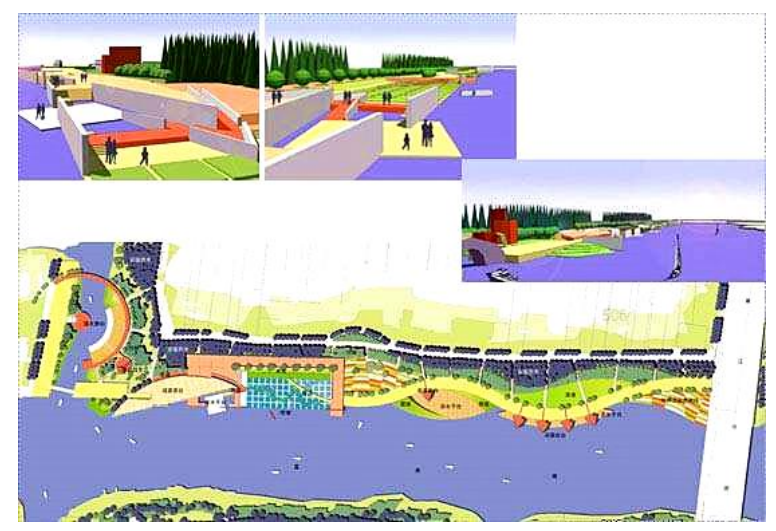

Fig 1: Examples of urban green landscape pattern

The key to realize the feature extraction of remote sensing information of urban greening is to deal with the spectral greening corridor partition of urban greening landscape pattern, which provides accurate data basis for the construction of urban landscape and landscape architecture. In urban landscape design, due to the aggregation of green patches and the complexity of the region, it is difficult to effectively extract the spectral characteristics of remote sensing information, which restricts the scientific planning of urban landscape design. Therefore, it is of practical significance to study the analysis of urban landscape pattern based on the combination of green patches. The schematic diagram of spectral green corridor zoning of urban green landscape is shown in Figure 2.

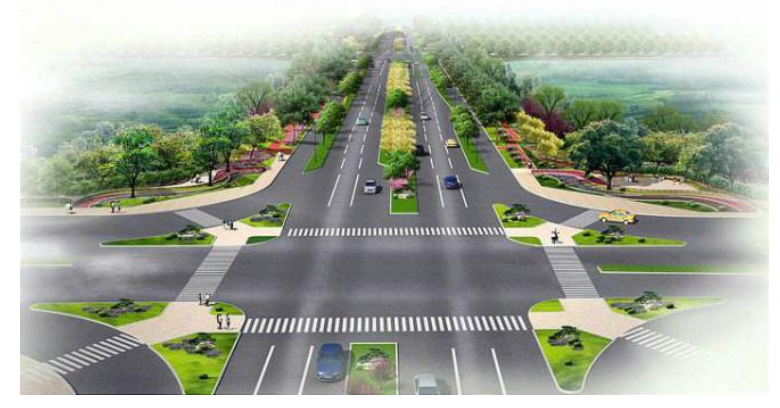

Fig 2: Schematic diagram of the spectral green corridor city green landscape partition

\section{Mathematical model construction of feature extraction and information extraction of urban green landscape}

Based on the above analysis of urban green landscape pattern, in order to achieve the spectral feature extraction of urban green landscape pattern. It is convenient for the expert system to make decision and construct the expert evaluation system for the analysis of urban landscape pattern, which needs to collect the spectral remote sensing characteristics of urban landscape pattern. The feature points of green spot feature information are extracted, and the spectral remote sensing collection formula of green landscape pattern is as follows [9-13]:

$$
\begin{aligned}
& \mathrm{y}=\overline{\mathrm{y}}+R_{\mathrm{i}} \mathrm{d} \\
& \mathrm{z}=\overline{\mathrm{z}}+R_{\mathrm{h}} \mathrm{d}
\end{aligned}
$$

In the above formula, $R_{t}$ represents the edge change pixel of green landscape structure, and $R_{h}$ is the change rate of green principle style texture. Using the expert evaluation system evaluation method to design the spectral remote sensing characteristic statistical model of green patches, the formula group of characteristic information evaluation coefficient is shown as follows:

ISSN: 0010-8189 


$$
\begin{aligned}
& \psi_{11}=P A+A^{T} P+Q_{1}+R_{1}+R_{2}+K_{1}+K_{1}^{T} \\
& \psi_{12}=W_{1}-K_{1}+M_{1}+K_{2}^{T} \\
& \psi_{13}=P B+L_{1}+M_{1}+K_{3}^{T} \\
& \psi_{14}=-L_{1}+K_{4}^{T} \\
& \psi_{15}=-W_{1}+K_{5}^{T} \\
& \psi_{23}=L_{2}-M_{2}+W_{3}^{T}-K_{3}^{T}+M_{3}^{T} \\
& \psi_{24}=-L_{2}+W_{4}^{T}-K_{4}^{T}+M_{4}^{T} \\
& \psi_{25}=-W_{2}+W_{5}^{T}-K_{5}^{T}+M_{5}^{T} \\
& \psi_{33}=-(1-\mu) Q_{2}+L_{3}-L_{3}^{T}-M_{3}-M_{3}^{T} \\
& \psi_{34}=-L_{3}+L_{4}^{T}-M_{4}^{T} \\
& \psi_{35}=-W_{3}+L_{5}^{T}-M_{5}^{T} \\
& \psi_{44}=-R_{1}-L_{4}-L_{4}^{T} \\
& \psi_{45}=-W_{4}-L_{5}^{T} \\
& \psi_{55}=-R_{2}-W_{5}-W_{5}^{T} \\
& U=h_{1} Z_{1}+h_{2} Z_{2}+h Z_{3}
\end{aligned}
$$

In the establishment of the relevant urban landscape geographic information system, access to provide a powerful means of information acquisition. On the pixel correlation $y=y_{i}+1$, there are two pixels $\mathrm{PE}_{1}$ and $\mathrm{pe}_{2}$ in the interval $\left(x_{i, r}-\frac{1}{2}, x_{i, r}-\frac{3}{2}\right)$. According to the principle of pixel point Association, when $\left(x_{i+1}, y_{i+1}\right)$ is to the right of the midpoint $\mathrm{M}\left(x_{i}+\frac{1}{2}, y_{i+1}\right)$, the pixel point $\mathrm{PE}_{1}$ is taken, otherwise it is the pixel point $\mathrm{PE}_{2}$, color adjustment and correction. In the random field of urban landscape analysis (RF), it can be expressed as the product of two independent random fields, that is, $C=\left\{C_{\mathrm{i}} ; i \in I\right\}$ in the partition of spectral green corridor. It can be expressed as:

$$
C=S \bullet \mu=\left\{S_{\mathrm{i}} \bullet U_{i}: i \in I\right\}
$$

For any $i$ and $j$ (i is not equal to $j$ ), $D_{i}$ is independent of $C_{j}$, and for a given $S_{i}, C_{i}$ and $D_{i}$ are independent of $S_{j}$. Because $C_{i}$ and $V_{i}$ obey the progressive rules of information extraction and extraction accuracy, the optimization equation of feature extraction of urban green landscape pattern is obtained as follows:

$$
I_{G S M}=I\left(C^{N} ; D^{N} \mid s^{N}\right)=\sum_{i=1}^{N} I\left(C_{i} ; D_{i} \mid s_{i}\right)
$$

Surf (speed up feature) is a fast robust feature extraction and registration algorithm based on SIFT (scale in variant feature transformation) algorithm. SIFT algorithm was proposed by Lowe in 1999. It is robust to image rotation, translation, zooming and denoising, but it takes a long time. Surf algorithm inherits the strong robustness of SIFT algorithm, and has high matching accuracy and fast speed. Therefore, surf algorithm is selected for UAV image registration. The flow chart of panorama registration algorithm is shown in Figure 3. 


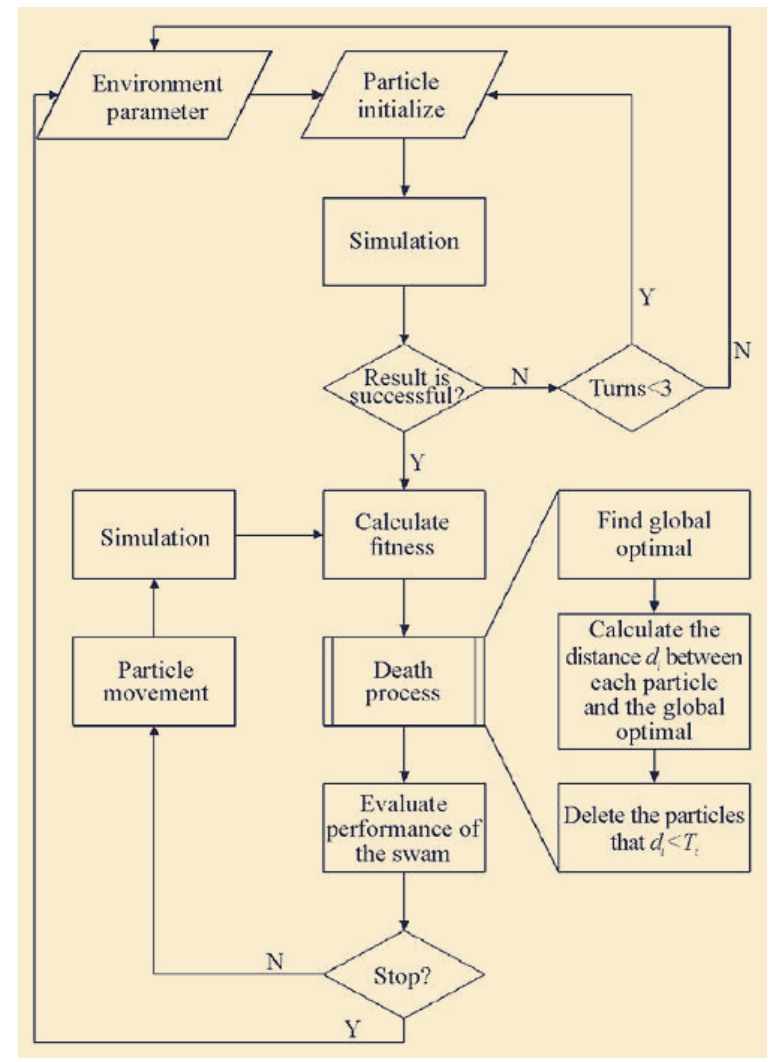

Fig 3: Algorithm flow chart

In order to improve the matching efficiency and ensure the scale invariance of the algorithm, the image must be layered first. The surf algorithm filters the integral image of the original image through different size box filters, thus forming different scale space. Then the Hessian matrix is used to detect the image extreme points on each layer of image, and the threshold value of Hessian matrix is set to filter the extreme points. Finally, the location and scale value of the feature points are obtained.

\section{Experiment result and analysis}

In order to verify the performance of the algorithm in the processing and feature extraction of spectral green corridor of landscape pattern combined with green patches from the perspective of simulation experiment, the simulation experiment is carried out. In the simulation experiment, image processing technology is used to classify the spectral feature information. On the basis of information extraction, the spectral remote sensing information of the urban green landscape pattern is compressed to realize the partition processing of the spectral green corridor of the urban green landscape pattern. The simulation test is based on the spectral image of a large city's urban green landscape pattern. The spectral image includes a large number of multi-source information such as vegetation and green landscape in the urban landscape. When the training vector size of image data is $256 \times 256$ $\times 224$ and the codebook size is 256 , the remote sensing spectral data collection method in this paper is used first, and the collected original data is shown in Figure 4.

Using the algorithm in this paper, the green patches are combined into the urban green landscape pattern, and the green corridor is divided into districts. Based on the combination of green patches and the collection of spectral spatial information characteristics, the simulation results are shown in Figure 5. 


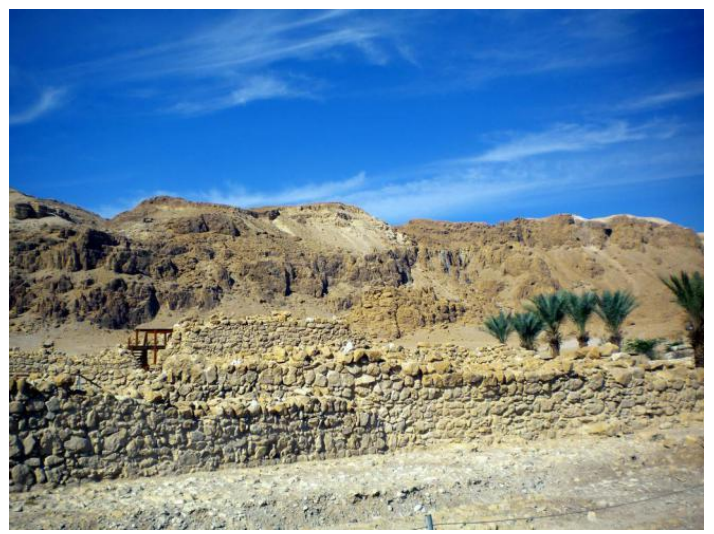

Fig 4: Original data acquisition

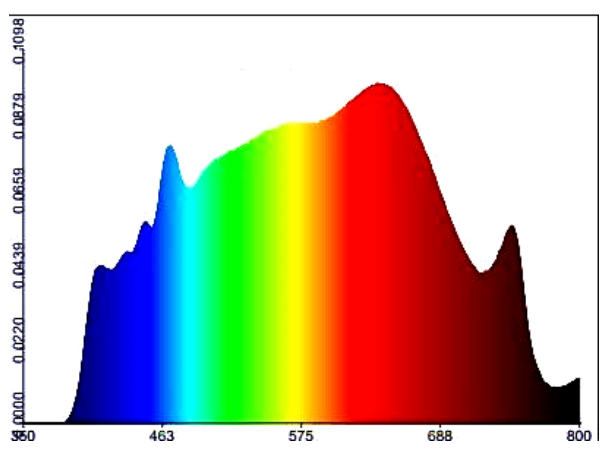

Fig 5: Green patches with collecting spectral spatial information feature

It can be seen from the analysis that the algorithm in this paper can effectively extract the features of the green corridor in the urban landscape and partition the corridor. The peak signal-to-noise ratio and root mean square error of the corridor partition are improved by $0.69 \mathrm{db}$ and $0.79 \mathrm{db}$ respectively compared with the literature algorithm, which shows the superior performance of the method in this paper. The research results can realize the extraction of remote sensing information characteristics of urban greening, and provide accurate data basis for the construction of urban landscape and landscape architecture.

The experimental data selected in this paper are UAV images in area a, with a height of $1000 \mathrm{~m}$, a number of image rows and columns of $5412 \times 7216$, a course overlap of $70 \%$ and a side overlap of $40 \%$. Most of the areas are houses and roads. Figure 6 shows the adjacent image of a certain navigation belt in A area, and Figure 7 shows 418 pairs of feature points extracted and roughly matched by surf algorithm. Figure 8 shows 274 pairs of feature points obtained by RANSAC purification and fine matching. The surf algorithm and RANSAC used in the experiment are all implemented by $\mathrm{C}$ language in Microsoft Visual $\mathrm{C}++6.0$ environment.

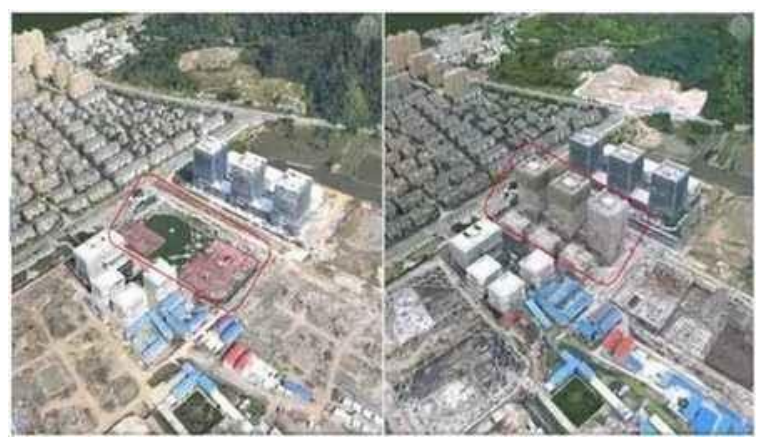

Fig 6: Image pairs of UAV in area A

ISSN: 0010-8189 


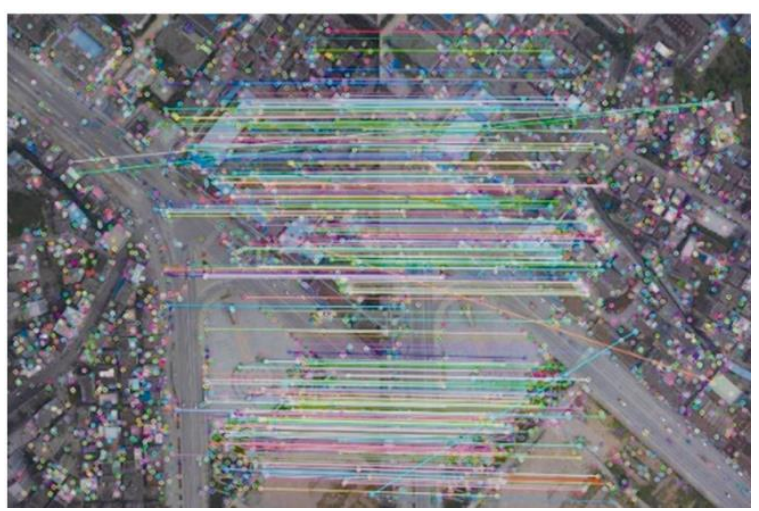

Fig 7: Rough match diagram

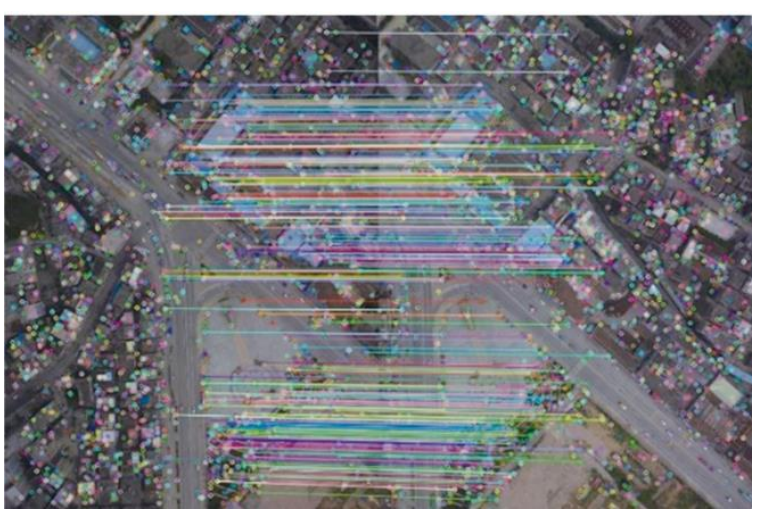

Fig 8: Fine match diagram

It can be seen that there are many houses in the image area, and the direction of houses in the city is basically the same, and the feature points extracted in the rough matching process are very similar, so there are a few wrong matching feature points. The fine matching eliminates the error points in the coarse matching and improves the robustness of the matching.

\section{Conclusion}

In the overall pattern planning of urban green landscape construction, it is necessary to solve the problem of feature quantification in the pattern analysis of urban green landscape and provide data support for urban landscape and landscape design. Through the spectral feature analysis of the pattern of urban green landscape, the green landscape corridor of urban ecological landscape is processed in different areas, which plays an extremely important role in urban ecological construction and environmental improvement. In this paper, surf algorithm is used for UAV image registration, which is divided into two steps: local registration and global registration. Experimental results show that the image registration algorithm based on surf features achieves better matching accuracy, effectively avoids the distortion in panorama, and finally gets a qualified and exquisite panoramic mosaic image of UAV. The algorithm provides accurate data basis for the construction of urban landscape and landscape architecture, and plays the advantages of strong unmanned flexibility and low cost. It provides a new method for earthquake prevention and disaster reduction, dynamic monitoring of land use.

\section{References}

[1] Lin Jun. Application of UAV Remote Sensing Technology in Campus Mapping. China Training, 2016, 10: 54-55

[2] Lu Jun. Discussion on the Application of Uav Aerial Photogrammetry in Topographic Mapping.

ISSN: 0010-8189 
Henan Building Materials, 2018 (04): 444-445

[3] Li Pingsheng. on the Application of Key Technologies of Uav Surveying and Mapping Data Processing. Scientific and Technological Innovation and Application, 2018, 254 (34): 153-154

[4] Wang Jun. Application of Small Uav in Large Scale Topographic Mapping. Housing and Real Estate, 2019, No. 543 (21): 186

[5] Xu Dibao, Huang Jian, Zheng Bin. Map Making of Surveying and Mapping Emergency Support Based on Low Altitude Uav. Chinese Scientific and Technological Achievements, 2018 (9): 32-35

[6] Zhan Hong. Research on the Application of Remote Sensing Aerial Survey Technology in Map Surveying and Mapping. Commodity and Quality, 2019, 000 (021): 98

[7] Zhang Ling. Application of Uav Aerial Photogrammetry in Topographic Mapping. Digital User, 2018, 024 (014): 255

[8] Huang Nan. Discussion on Intelligent Mapping Uav Mapping. Urban Construction Theory Research: Electronic Edition, 2012, 2: 1-6

[9] Zhen Ge. Application Analysis of Uav Aerial Survey in Large Scale Topographic Mapping. Geological and Mineral Mapping, 2019, 2 (4): 11-14

[10] He Yu. Application of Aerial Photogrammetry in Urban Surveying and Mapping. Global Human Geography, 2017, 2: 98

[11] Mit Develops Uav Search and Rescue System Without Gps Assistance. Radio Engineering, 2019 (4): $331-331$

[12] Sun Lei. Analysis on the Application of Uav Survey Technology in Topographic Survey. Architectural Engineering Technology and Design, 2016, 36 (18): 13-18

[13] Wang Chenjie, Luo Bin, Li Chengyuan. Cooperative Mapping and Navigation of Uav Vision Slam. Journal of Surveying and Mapping, 2020, V.49 (06): 101-110 